\title{
DEPRESIÓN Y FACTORES DE RIESGO EN EL ADULTO MAYOR, HOSPITAL SANTA MARÍA DEL SOCORRO DE ICA, 2016
}

\author{
Depression and risk factors in the elderly, Santa Maria of the Socorro of Ica Hospital, 2016
}

\author{
Dra. Zonia Felicitas Quispe Quispe, 2,a,b, Nut. Yolanda Chinarro de Pun ${ }^{1, d}$, Lic. Enf. Ana Cecilia Flores \\ García $^{2, a, c}$, Lic. Enf. Betsy Herencia Gabulle,2,a
}

\author{
${ }^{1}$ Facultad de Enfermería de la Universidad Nacional San Luis Gonzaga de Ica \\ ${ }^{2}$ Hospital Santa María del Socorro de Ica. \\ a Licenciada en Enfermería, ${ }^{b}$ Doctor en Salud Pública, 'Magíster en Educación; ' Nutricionista
}

\section{RESUMEN}

Objetivo: Determinar la relación entre la depresión y los factores de riesgo, en adultos mayores de 65 años hospitalizados en el Hospital Santa María del Socorro de Ica, 2016. Material y métodos: Estudio descriptivo, correlacional, no experimental, de diseño transversal, muestra intencional de 111 adultos mayores de 65 a 80 años, de enero a marzo del 2016 en los servicios de medicina (81 pacientes) y cirugía (30 pacientes); se utilizaron los instrumentos de Yesavage y Folstein, con alta validez y confiabilidad Resultados: $11,71 \%$ de los adultos mayores tienen depresión severa, 58,6\% están con depresión leve, y 29,7\% no están deprimidos. La depresión se relaciona en forma directa con los factores de riesgo como la edad $r, 597 p, 000$ y la condición laboral $r, 487 p, 000$ e inversamente con el sexo $r-, 350 p, 000$ la dependencia $r-, 463 p, 000$ el aspecto social y los ingresos económicos $r-, 359 \mathrm{p}, 000$. . La depresión severa está presente en mayor proporción en los hombres (9 pacientes) que en las mujeres (4 pacientes). $88,2 \%$ de los adultos mayores no presentan signos de demencia, 10,8\% tienen probabilidad de adquirir demencia. Se ha encontrado 1 caso $(0,9 \%)$ de demencia. Conclusiones: $11,71 \%$ de los adultos mayores tienen depresión severa; Hay relación estadística significativa directa positiva entre la depresión y los factores de riesgo a excepción del estado civil. Se recomienda intervenir a los adultos mayores detectados con depresión severa, asegurándoles el tratamiento especializado psicológico, psiquiátrico, y medicamentoso, así como también la necesidad de incluir tratamientos basados en la evidencia científica en Asistencia Primaria, el cual pueden reducir los costos sociales y sanitarios.

Palabras clave: Depresión, adultos mayores, factores de riesgo.

\section{SUMMARY}

Objective: To determine the relationship between depression and risk factors in adults older than 65 years hospitalized at the Santa Maria Hospital Relief Ica, 2016. Material and Methods: Descriptive, correlational, not experimental, cross design, purposive sample of 111 adults aged 65 to 80 years, from January to March 2016 medical services (81 patients) and surgery (30); Yesavage and Folstein instruments were used, with high validity and reliability. Results: $11.71 \%$ of older adults have severe depression, 58.6\% are mildly depressed, and $29.7 \%$ are not depressed. Depression is associated directly with risk factors such as age r, p 597, 000 and working condition $r, p$ 487, 000. And conversely with sex $r-, p$ 350, 000 dependence $r-p$ 463, 000 the social aspect and income $r-, p 359,000$. The Severe depression is present in greater proportion in men (9 patients) than in women (4 patients). $88.2 \%$ of the elderly have no signs of dementia, $10.8 \%$ have a chance of getting dementia. There is 1 case (0.9\%) of dementia. Conclusions: $11.71 \%$ of older adults have severe depression; There are significant positive direct statistical relationship between depression and risk factors except marital status. It is recommended to intervene older adults identified with severe depression, assuring the psychological, psychiatric and drug treatment as 
well as the need to include based on scientific evidence in primary care, which can reduce the social and health costs treatments.

Keywords: Depression in older adults, depression and risk factors.

\section{INTRODUCCIÓN.}

La depresión es un problema de salud pública y un predictor importante de discapacidad funcional y mortalidad, con consecuencias económicas anuales estimadas en 118 billones de euros en Europa y de 83 mil millones de dólares en los EE.UU. Esta enfermedad contribuye a una disminución en la calidad de vida, disminución funcional, discapacidad marcada y un incremento en la utilización de servicios, así como incremento en la mortalidad por comorbilidad con otras condiciones médicas (1).

Los síntomas de la depresión en el adulto mayor se pueden manifestar de formas muy variadas, desde sentimientos de inutilidad y tristeza, desánimo, hasta la afectación del desarrollo de la vida cotidiana (2). Algunos de los síntomas emocionales de la depresión son: alteraciones del humor o del estado de ánimo, disminución de la capacidad para disfrutar de eventos familiares y sociales, pérdida de interés por las cosas, disminución de la vitalidad laboral y social. Sentimientos de culpa, pesimismo, autoreproches, ideas de suicidio, dificultad para concentrarse, desesperanza y otros (3).

Entre los síntomas físicos se pueden mencionar: cefalea, problemas gastrointestinales, cansancio o falta de energía, pérdida de peso, falta de apetito, hipersomnia (trastorno del sueño caracterizado por somnolencia), vértigo (sensación de falta de equilibrio), etc. (4).

También ciertos factores pueden incidir en la depresión del adulto mayor, tales como: las enfermedades crónicas, pérdidas de seres queridos, disminución de la capacidad económica (pensión económica insuficiente), perdida de roles, cambio en las actividades físicas y de relaciones sociales, deterioro de la salud que en algunos casos conlleva a la dependencia física y disminución de la autonomía (5).

Los trastornos depresivos en adultos mayores tienen connotaciones negativas en su vida, estudios realizados señalan que los adultos mayores deprimidos están en riesgo de incrementar enfermedades cardiacas o de muerte, respecto de los no deprimidos (6).

Una de las enfermedades mentales que se presentan en la población a nivel mundial es la depresión (7), tiene orígenes multicausales, los cuales interaccionan de manera agrupada o conjunta (8).

La Organización Mundial de la Salud (OMS) nos informa que la proporción de personas mayores está aumentando rápidamente en todo el mundo. Según se calcula, entre 2000 y 2050 dicha proporción pasará de 11\% a $22 \%$. En números absolutos, el aumento previsto es de 605 millones a 2000 millones de personas mayores de 60 años. Los adultos mayores pueden sufrir problemas físicos y mentales que es preciso reconocer (9).

En Estados Unidos y Europa la prevalencia de depresión oscila entre el $6 \%$ y el $20 \%$, aunque en Italia asciende hasta el $40 \%$, en México, hasta el 2005, la prevalencia fue de $5.8 \%$ en las mujeres y $2.5 \%$ en los hombres, incrementándose con la edad. Sin embargo, otros estudios reportan que este es el trastorno afectivo más frecuente en personas mayores de 60 años, esto es en un 15 a $20 \%$, en la población ambulatoria, incrementándose hasta en 25 a $40 \%$ en la hospitalizada; y la incidencia de casos nuevos por años es de aproximadamente15\%(10).

Según el INEI, en el Perú el grupo de personas de 65 y más años de edad, se 
incrementará sostenidamente en las próximas décadas. De poco menos de 1,5 millones de adultos mayores en el 2010, se pasará a casi 6,5 millones en 2050; la mayor parte de los cuales serán mujeres. Este proceso de envejecimiento demográfico plantea desafíos y exigencias de diferentes naturalezas en términos de políticas y programas sociales a nivel regional y local. CEPAL considera que para la región de América latina la población adulta mayor, pasó de apenas un 5,6\% del total regional en 1950 a un $10 \%$ en el año 2010. En el contexto de América latina, el Perú registra un crecimiento de población adulta mayor de $8,76 \%$ al año 2010(11).

Estudios epidemiológicos recientes realizados en el Perú por el Instituto Especializado de Salud Mental Honorio Delgado - Hideyo Noguchi confirman los hallazgos de otros países: hasta el $37 \%$ de la población tiene la probabilidad de sufrir un trastorno mental alguna vez en su vida. La prevalencia de depresión en Lima Metropolitana es de $18,2 \%$, en la sierra peruana de $16,2 \%$ y en la Selva de 21,4\%(12). En Ica, Gamonal Aranibar (2008), encontró elevada prevalencia de depresión en adultos mayores (53,7\%), asociada a la edad, y escolaridad, índice que es necesario corroborarlo (13).

En base a lo anteriormente expuesto, la investigación determina la relación entre la depresión, y los factores de riesgo, en adultos mayores de 65 años en el Hospital Santa María del Socorro de Ica, en el año 2016, justificando su desarrollo, por el incremento de casos de adultos mayores con altos niveles de depresión que se atienden en dicho nosocomio, impactando en su salud general.

\section{MATERIAL Y MÉTODOS.}

El estudio fue ejecutado considerando los principios éticos de autonomía, beneficencia y justicia, siendo el tipo de investigación no experimental, sin manipulación de la variable independiente, descriptiva- correlacional, de diseño transversal, que analiza las variables: Depresión y factores de riesgo.

La muestra fue intencional, la constituyeron el $100 \%$ de la población, es decir, los adultos mayores de 65 a 80 años de edad, hospitalizados, en los servicios de Medicina y Cirugía del Hospital Santa María del Socorro de Ica, durante los meses de enero a marzo del 2016, fueron 81 pacientes del servicio de medicina y 30 del servicio de cirugía, haciendo un total de 111 pacientes.

Se utilizaron como técnicas: La entrevista, la observación, el análisis y la síntesis.

Utilizamos dos instrumentos para recoger la información:

Escala de Yesavage: En su versión original de 30 items, con la que se valora la no depresión (0 a 10 puntos), depresión levemoderada (11 a 20 puntos), y depresión severa (mayor de 20 puntos). Las respuestas esperadas están en escala dicotómica de si- no, y valen un punto cada respuesta afirmativa.

Mini examen cognoscitivo de Folstein: Versión de Lobo, con la cual se determinó la demencia (corte 20 puntos), probabilidad de demencia=14 a 19 puntos, demencia $=0$ a 13 puntos; no presencia de demencia $=20$ a 35 puntos.

\section{RESULTADOS.}

En la tabla 1 se observa que la prevalencia de la depresión severa es de $11,71 \%$, los resultados indican además la presencia de depresión leve en $58,6 \%$ de los adultos mayores. El 29,7\% no presentan signos o síntomas de depresión, es decir no están deprimidos, según la escala de YESAVAGE.

Tabla 1. Depresión según escala YESAVAGE en los adultos mayores, Hospital Santa María del Socorro Ica 2016

\begin{tabular}{lcc}
\hline \multicolumn{1}{c}{ Escala YESAVAGE } & f & $\%$ \\
\hline No deprimido $(0-10)$ & 33 & 29,7 \\
Depresión leve $(11-20)$ & 65 & 58,6 \\
Depresión severa $(21-30)$ & 13 & 11,7 \\
\hline Total & 111 & $100 \%$ \\
\hline
\end{tabular}


En la tabla 2 se observa que $88,2 \%$ de los adultos mayores no presentan signos de demencia, 10,8\% tienen probabilidad de adquirir demencia. Se ha encontrado 1 caso $(0,9 \%)$ de demencia.

Tabla 2. Demencia según escala FOLSTEIN en los adultos mayores, Hospital Santa María del Socorro Ica 2016

\begin{tabular}{|lcc|}
\hline \multicolumn{1}{c}{ Escala FOLSTEIN } & $\mathbf{f}$ & $\%$ \\
\hline Demencia & 1 & 0,9 \\
Probable demencia & 12 & 10,8 \\
No demencia & 98 & 88,2 \\
\hline Total & 111 & $100 \%$ \\
\hline
\end{tabular}

Se ha encontrado relación estadística significativa directa positiva entre la depresión con la edad $r, 597 \mathrm{p}, 000$ y la condición laboral $r, 487 p, 000$. Así mismo se ha determinado relación estadística inversa entre la depresión con el sexo $r$-,350 p ,000 la dependencia $r-, 463 \mathrm{p}, 000$ el aspecto social (viven en compañía) y los ingresos económicos $r-, 359 p, 000$

\section{DISCUSIÓN.}

La depresión mayor es uno de los trastornos psicológicos más prevalentes y que más problemas de discapacidad genera en la actualidad. (Cano y col 2012) (14). En el estudio se ha encontrado depresión severa en $11,71 \%$ de los adultos mayores, así misma depresión leve en $58,6 \%$, y $29,7 \%$ están normales, no tienen síntomas o signos de depresión. Estos resultados son aproximados al de Oscar Medina (2013) (15), quien encontró $10 \%$ de depresión severa en adultos mayores, también se contextualiza en el estudio de Cano Vindel y col (2012) (14), en España, quien señala que la prevalencia año de depresión en asistencia primaria (AP), es de $9,6 \%$ a $20,2 \%$. Contrastan en cambio con Friedrich von Mühlenbrock, Rocío Gómez D, Matías González V, Alberto Rojas A, Lorena Vargas y Christian von Mühlenbrock P (2011) (16) quienes, en pacientes mayores de 60 años hospitalizados en el servicio de medicina, encontraron prevalencia de depresión en
$22,13 \%$ de ellos, de las cuales $28,12 \%$ en mujeres y $16,41 \%$ en hombres; con Crespo Vallejo Jonathan Raúl (2011) (17), de Ecuador, quien en casa de reposo hallo $70 \%$ de adultos mayores con depresión. Así mismo con Martínez Mendoza J, Martínez Ordaz V, Esquivel Molina C, Velazco Rodríguez V (2007)(18), quienes también con adultos mayores hospitalizados, mayores de 65 años, encontraron 3,1\% con demencia, $50 \%$ con depresión, Pilar Guallar-Castillóna, y col (2006)(19), en España, con pacientes mayores de 65 años, encontraron 48,5\% con depresión, con mayor incidencia en mujeres, González Marilú, Robles Esther (2011)(20) en Lima con adultos mayores, encontró riesgo moderado de depresión. Los adultos mayores del grupo etario de 60 a 80 años tenían mayor riesgo de depresión que los adultos de mayor edad, Juárez José M; León Angélica F; y Alata Linares Vicky (2010)(21), en Lima - Perú, con adultos mayores de 60 años, en el AA.HH Viña alta de la Molina, encontraron alta prevalencia de depresión en algún grado, comparado con el $9,8 \%$ para la población adulta mayor de Lima metropolitana, con Gamonal Aranibar, Oshin Elsy; Delgadillo Soriano, Alexander; Antonio Llerena, Fredy Donato (2009)(22) quienes en Ica, encontraron $53,7 \%$ de depresión, $43 \%$ depresión leve-moderada y $10,7 \%$ de depresión severa, resultado similar al encontrado en el presente estudio(11,71\%). Todos estos estudios fueron realizados con el instrumento de Yesavage. Las diferencias de los resultados encontrados con los diversos estudios mencionados, se explican no tanto por la metodología aplicada, si no por los factores socioeconómicos, culturales, ambientales, sociológicos, y otros que se relacionan de alguna manera con la depresión. Al respecto Cano (2012) (14) señala que la depresión posee una alta comorbilidad cursando con otros problemas tanto físicos como psicológicos, y sugiere la necesidad de incluir tratamientos basados en la evidencia científica en AP, los cuales según Cano han demostrado ser más eficaces que la práctica habitual y que 
pueden reducir los costes sociales y sanitarios.

\section{CONCLUSIONES.}

1. Hay relación estadística significativa directa positiva entre la depresión con la edad, y la condición laboral, y relación inversa entre la depresión con el sexo, la dependencia, el aspecto social, y los ingresos económicos.

2. $11,71 \%$ de los adultos mayores tienen depresión severa, 58,6\% leve, y 29,7\% no están deprimidos. El $88,2 \%$ de los adultos mayores no presentan signos de demencia, y 10,8\% tienen probabilidad de adquirirla. Se ha encontrado 1 caso $(0,9 \%)$ de demencia.

3. $54,1 \%$ de la población tiene entre 65 a 70 años de edad, $52,3 \%$ son hombres, $59,5 \%$ no dependen de sus hijos o familiares, 92,8\% viven en compañía de su pareja, o hijos, o familiares, 59,5\% son casados, $7,2 \%$ tienen diabetes mellitus, 4,5\% HTA, 4,55 artritis, 54,1\% reciben pensión como cesantes, 0 jubilados; $80 \%$ tienen ingresos económicos entre 400 a 800 soles mensuales.

\section{RECOMENDACIONES}

1. La Jefatura del servicio de Psicología, debe intervenir al $11,71 \%$ de los adultos mayores detectados con depresión severa, asegurándoles un tratamiento especializado, psicológico, psiquiátrico, y medicamentoso.

2. La Dirección del Hospital debe gestionar tratamientos basados en la evidencia científica, en Asistencia Primaria, que han demostrado ser más eficaces que la práctica habitual y que pueden reducir costos sociales y sanitarios-

3. Los investigadores pueden continuar con la investigación extendiendo a los asilos, casas de reposo, y a la población adulta mayor de la localidad y el país.

\section{LIMITACIONES DEL ESTUDIO}

El estudio no tuvo limitaciones, desarrollándose exclusivamente en adultos mayores, con presupuesto, metodología y tiempo de ejecución en forma apropiada.

\section{CORRESPONDENCIA:}

Dra. Zonia Felicitas Quispe Quispe

Correo electrónico:

zoniaquispe@hotmail.com

Celular: +51956004215

\section{REFERENCIAS BIBLIOGRÁFICAS.}

1. Mori N, Caballero J. Historia natural de la depresión. Rev. Peruana de Epidemiología. 2010. (Internet) Lima (Citado el 24 de setiembre 2015) Disponible en: http://www.redalyc.org/articulo.oa?id= $\underline{203119666002}$

2. Sánchez Y, Castañeda H. Prevalencia de depresión en Adultos Mayores de 65 Años y Propuesta de Intervención Preventiva. Geroinfo: Publicación de Gerontología y Psiquiatría. 2008, v.3, n.3, pp.4-5.

3. Oquendo E, Soublet 0 . ¿Es la depresión un problema de salud en un hogar del adulto mayor? Rev. Cubana de Enfermería. 2003; 19(.2): 1-6.

4. Marsiglia G. Depresión: Visión holística de la medicina interna.Rev. Gaceta Médica de Caracas. 2008; .116 (1): 1-10.

5. Lizondo G, Llanque C. Depresión. Rev. Paceña de Medicina Familiar. 2008; 5(.7):.24-31.

6. Trinidad, M., Valenzuela, E., Marín, P. Depresión en el adulto mayor: evaluación preliminar de la efectividad, como instrumento de tamizaje, de la versión de 5 ítems de la Escala de Depresión Geriátrica. Rev Médica de Chile, 2000; 128 (11):.2-3. 
7. Ayllón A, Guadarrama G, Márquez 0 . Depresión: estudio comparativo en adultos mayores asistentes y no asistentes a los clubes del DIF de la ciudad de México. Rev Mexicana de investigación en Psicología. 2012; 4 (1): 58-66.

8. Martínez, A, Martínez, O., Esquivel, G., Velasco, M. Prevalencia de depresión y factores de riesgo en el adulto mayor hospitalizado. Rev Médica Instituto México Seguro social. 2005; 45 (1): 21 28.

9. Organización Mundial de la Salud (OMS). Datos y estadísticas. La salud mental y los adultos mayores. 2013. (Internet) (Citado el 12 de Agosto 2015) Disponible en: http://www.who.int/media centre/factsheets/fs381/es/

10. Bello M, Puentes R, Medina M, Lozano R. Prevalencia y diagnóstico de depresión en población adulta en México. Salud Pública México. 2005; 47. Suplemento 1.

11. Ministerio de la Mujer y Población Vulnerable (MIMP). Cuaderno Sobre Población Vulnerable N5. 2013. (Internet) ( Citado el 17 de Setiembre 2015) Disponible en: http://www.mimp.gob.pe /files/mimp/especializados/boletines dv $\underline{\mathrm{mpv} / \mathrm{cu} \text { aderno } 05 \mathrm{dvmpv} . p \mathrm{df}}$

12. Rondón, M. Salud mental: un problema de salud pública en el Perú. Rev. Perú. Med. Exp. Salud Pública (online) 2006;23(4): 237-238. (Citado el 24 Setiembre 2015).

13. Gamonal O, Delgadillo A, Antonio F. Prevalencia y factores asociados a depresión en adultos mayores de la provincia de Ica. 2008. Tesis Pregrado. Ica, Perú Facultad de Medicina Humana. Universidad Nacional San Luis Gonzaga de Ica; 2009.
14. Cano A, Salguero J, Maewood C, Dongi E, Latorre J. La depresión en atención primaria prevalencia, diagnóstico y tratamiento. Rev. Papeles del Psicólogo, España 2012; 33 (1).

15. Medina O. Prevalencia de la depresión en adultos mayores institucionalizados de la ciudad de Medellín, Colombia ;2013.

16. Von Mühlenbrock $F$, Gómez $R$, González M, Rojas A, Vargas L, Von Mühlenbrock C. Prevalencia de depresión en pacientes mayores de 60 años hospitalizados en el servicio de medicina interna del hospital militar de Santiago de Chile. Rev Chil NeuroPsiquiat 2011; 49 (4): 331-33

17. Crespo J. Prevalencia de depresión en adultos mayores de los asilos de los cantones Azogues, Cañar, Tambo y Délegacion de la provincia del Cañar. Ecuador ;2011.

18. Martínez J, Martínez V, Esquivel C, Velasco V. Prevalencia de depresión y factores de riesgo en el adulto mayor hospitalizado. Rev. Médica Instituto México Seguro social. 2007; 45 (1):2128. Internet) México .(Citado el 16 de Setiembre 2016) Disponible en: http://edumed.imss.gob.mx/edumed/rev $\mathrm{med} / \mathrm{pdf} / \mathrm{gra}$ art/A91.pdf

19. Guallar P, Magariños M, Montoto $C$, Tabuencaa A, Rodríguez $\mathrm{C}$, et al. Prevalencia de depresión, y factores biomédicos y psicosociales asociados, en ancianos hospitalizados con insuficiencia cardiaca en España; 2006

20. Gonzáles M, Robles J. Riesgo de depresión del adulto mayor según test de Yesavage en el Centro Residencial Rodulfa Viuda de Canevaro, Tesis Pregrado. Lima, Perú. Universidad Wiener; 2011 
21. Juárez, J., León, A., Alata, V. Evaluación del grado de depresión en adultos mayores de 60 años del AA. HH "Viña Alta" Lima- Perú. Rev. Horizonte Médico.2012; 12(.2):.28-29. (Internet). Perú: (Citado 12 de Agosto 2015) Disponible en:

http://www.medicina.usmp.edu.pe/ horizonte/2012 II/Art4 Vol12 N2.pdf.
22. Gamonal O, Delgadillo A, Antonio F. Prevalencia y factores asociados a depresión en adultos mayores de la provincia de Ica. 2008. Tesis Pregrado. Ica, Perú. Facultad de Medicina Humana. Universidad Nacional San Luis Gonzaga de Ica. 2009.

Recibido: 12/12/2020

Aprobado para publicación: 09/04/2021 